\title{
Ausstellungskonzept \\ Militärgeschichte ausstellen. Neue konzeptionelle Ansätze am \\ Militärhistorischen Museum Flugplatz Berlin-Gatow
}

Von DoRis MülLer-ToOveY

Das Militärhistorische Museum Flugplatz Berlin-Gatow (MHM Gatow) ist seit 2011 eine Zweigstelle des Militärhistorischen Museums in Dresden (MHM Dresden). Es ist aus einer bereits in den 1950er Jahren begründeten privaten Sammlung hervorgegangen, die sich bis Anfang der 1990er Jahre in Hamburg-Appen befand. 1987 kam die damals als „Luftwaffenmuseum“ bezeichnete Einrichtung in die Trägerschaft der Bundeswehr und zog 1994 an den jetzigen Standort, auf den ehemaligen Flugplatz Berlin-Gatow, dessen Geschichte als authentischer historischer Ort den Bogen von der geheimen Aufrüstung in den 1930er Jahren über die Blockkonfrontation im Kalten Krieg bis zur Wiedervereinigung spannt. Damit ist das Flächendenkmal das „erste Exponat" des Museums und die Standortgeschichte integraler Bestandteil der musealen Präsentation. Dass die aus den 1930er Jahren stammende Bausubstanz dringend sanierungsbedürftig ist, wurde bereits zum Zeitpunkt der Übernahme der Liegenschaft erkannt. Der damalige Leiter der Abteilung Ausstellungen verantwortete die erste inhaltliche Rahmenkonzeption. Doch aus verschiedenen Gründen konnte das Projekt danach nicht in Angriff genommen werden. 2016/17 kam wieder Bewegung in die Angelegenheit: Das Bundesministerium für Verteidigung sprach sich dezidiert für die Sanierung und Neukonzeption in Gatow aus und am Museum konnte eine Stelle in der Abteilung Neukonzeption besetzt werden. Ein Team des Museums arbeitet mit Unterstützung durch zwei externe Fachwissenschaftler bereits an der inhaltlichen Weiterentwicklung des Museums, auch wenn der infrastrukturelle Planungsprozess noch in der Prüfung ist und der Zeitplan für die Umsetzung noch nicht feststeht.

Der folgende Beitrag ist aus diesem Grund als Werkstattbericht zu verstehen. Er gibt einen Einblick in den am Museum laufenden inhaltlichen Diskurs über die konzeptionelle Weiterentwicklung und teils auch Neuausrichtung des Museums. Dazu gehören auch Fragen zur Präsentation insbesondere der militärischen Großtechnik und nach der Rolle, die das Museum im Erinnerungsdiskurs zu Krieg, Technik, Politik und Gesellschaft spielen soll. 


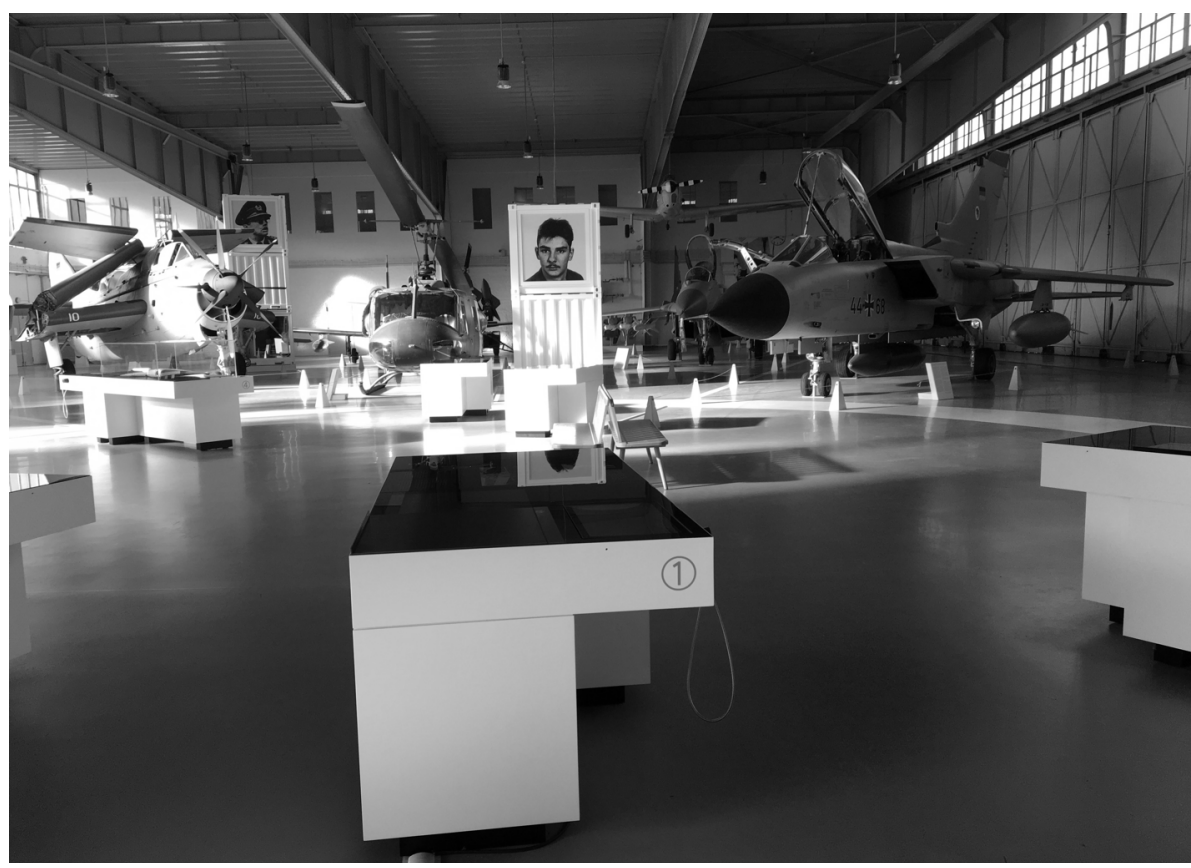

Abb. 1: Blick in die aktuelle Dauerausstellung „Zwischenlandung“ des MHM Gatow. (C) Militärhistorisches Museum Flugplatz Berlin-Gatow

\section{Militärgeschichte und Militärtechnik im Museum - ein Exkurs}

1987 schrieb der Technikhistoriker Kurt Möser: „Daß Militärtechnik und -geschichte heute, um es vorsichtig auszudrücken, ambivalente Urteile hervorruft, ist angesichts des anhaltenden atomaren Wettrüstens, permanenter kriegerischer Konflikte und zweier verheerender Weltkriege nicht weiter verwunderlich. Für viele hat allein schon die Beschäftigung mit dem Thema Krieg und Militär Tabucharakter oder gar schon einen obszönen touch bekommen. " Seither hat sich einiges geändert. Mit der deutschen Wiedervereinigung, dem Ende der Blockkonfrontation, der Entwicklung neuer Krisenherde und den Erfahrungen einer „Armee im Einsatz“ wurde in der Bundesrepublik der militärische Einsatz als Instrument der Politik wieder denkbar und Militärgeschichte salonfähig. Es gibt in Deutschland mittlerweile eine kritische und integrative Militärgeschichte, die politische, soziologische und wirtschaftliche Fragestellungen einbezieht und auch das handelnde Individuum in den Blick nimmt.

Auch die museale Präsentation von Militärtechnik und Militärgeschichte hat sich entsprechend verändert. Im Dezember 2008 veranstaltete die Koordinationsstelle des Projektverbundes Zeitgeschichte Berlin-Brandenburg

1 Kurt Möser, Militärgeschichte und -technik im Technikmuseum, in: Kritische Berichte 15, 1987, H. 3/4, S. 66-75, hier S. 66. 
einen Workshop zum Thema „Krieg und Gewalt ausstellen“, bei dem es um folgende Fragen ging: Wie lässt sich Gewalt überhaupt „ausstellen“? Welche ethischen Leitlinien sollen Kriegs- und Gewaltausstellungen zugrunde liegen? Ist ein szenisch-emotionaler oder ein dokumentarisch-sachlicher Zugang besser geeignet? Wie kann die Faszination der ausgestellten Waffen gebrochen werden? Damit waren die wesentlichen Probleme und Fragestellungen benannt, die nach wie vor für alle, die Militärgeschichte und Militärtechnik ausstellen, relevant sind. Militärische und militärtechnische Exponate können noch weniger als jedes andere historische Exponat auf eine Kontextualisierung verzichten. Erst durch die Art der Präsentation und den mitgelieferten Kontext werden sie zum Sprechen gebracht - und können dabei ganz unterschiedliche Geschichten erzählen. Insbesondere militärtechnische Exponate sind in ihrer Komplexität nicht zu verstehen, wenn sie lediglich „neutral“, mit technischen Daten versehen, präsentiert werden.

Was die Verbindung des Ansatzes einer integrativen Militärgeschichte mit dem Blick auf einzelne Akteure betrifft, hat das MHM Dresden bereits erfolgreich neue Wege eingeschlagen und greift in seinen Ausstellungen dezidiert soziale, politische und wirtschaftliche Fragestellungen auf. ${ }^{2}$ Diesem Ansatz fühlt sich auch das MHM Gatow verpflichtet. Gemeinsam ist beiden Häusern zudem der Fokus auf die deutsche Geschichte - dies immer in Verbindung mit internationalen Bezügen. Unterschiede ergeben sich aus der grundsätzlichen Aufgabenstellung der Museen. Das MHM Dresden ist thematisch breiter aufgestellt und folgt einem kulturwissenschaftlichen Ansatz, der die Frage nach der menschlichen Gewalt als anthropologischer Grundkonstante ins Zentrum stellt. Das MHM Gatow konzentriert sich auf das Thema militärische Luftfahrt und Luftkrieg, nimmt dabei stärker militärtechnologische Entwicklungen mit ihren vielfältigen Kontexten in den Blick und berücksichtigt politikwissenschaftliche Ansätze. Dies vor dem Hintergrund, dass insbesondere die Bedrohung aus der Luft das außenpolitische Handeln, Militärstrategien, Kriegsführung und nicht zuletzt unsere Wahrnehmung von Krieg nachhaltig verändert hat.

\section{Konzeptionelle Grundzüge - Konzentration und Vielfalt}

Nach wie vor ist es in Deutschland politisch-gesellschaftlicher Konsens, gegenüber allem Militärischen einen - im Vergleich zu anderen Ländern kritisch-zurückhaltenden Umgang zu pflegen. Eine angesichts der deutschen Vergangenheit verständliche und legitime Haltung, die vor allem erinnerungs-

2 Gorch Pieken u. Matthias Rogg (Hg.), Militärhistorisches Museum der Bundeswehr. Ausstellung und Architektur, Dresden 2011; dies. (Hg.), Das Militärhistorische Museum der Bundeswehr. Ausstellungsführer, Dresden 2011; Gorch Pieken, Contents and Space. New Concept and New Building of the Militärhistorisches Museum of the Bundeswehr, in: Wolfgang Muchitsch (Hg.), Does War Belong in Museums? The Representation of Violence in Exhibitions, Bielefeld 2013, S. 63-82. 
kulturelle Gründe hat, denn die aus Nationalsozialismus und Vernichtungskrieg gezogenen Lehren sind für den Umgang mit den Themen Krieg und staatliche Gewalt in Deutschland zu Recht weiterhin grundlegend. Das steht auch nicht im Widerspruch zu der Forderung, dass sich die deutsche Gesellschaft und jeder Einzelne in der mittlerweile ihren 70. Geburtstag feiernden parlamentarischen Demokratie offen mit Fragen nach den Rahmenbedingungen und der Notwendigkeit militärischer Einsätze als Instrument staatlicher Gewalt und der damit verbundenen Verantwortung auseinandersetzen sollte. Vor dem Hintergrund der weltpolitischen Entwicklungen und sicherheitspolitischen Herausforderungen in Gegenwart und Zukunft erscheint dies von besonderer Relevanz. Das Wissen über die Gründe, warum Militärtechnologien entwickelt, produziert und wofür sie eingesetzt wurden und werden, unterstützt den notwendigen Diskurs zu den oben genannten Fragen. Dazu beizutragen ist eine wichtige Aufgabe des Museums.

Die Materie ist freilich hochkomplex und verlangt vom Museum nicht nur die sowohl ansprechende als auch substanzielle Vermittlung von Inhalten, sondern auch, die Besucher/innen zur eigenen Urteilsbildung zu befähigen. Sie kommen mit ihren eigenen sozialen, politischen und sehr persönlichen Hintergründen, haben unterschiedliche Erwartungen, Interessen, Vorkenntnisse. Es reicht daher nicht, historische Entwicklungen als eine Chronologie von Ereignissen nachzuerzählen. Anstatt zu beschreiben „wie“ etwas war, soll das Ziel sein, zu erklären, ,warum“ "etwas so war. Dafür werden verschiedene Ansätze, Methoden und Ebenen der inhaltlichen Vermittlung diskutiert, wie die Reduktion der Einzelthemen zugunsten ihrer vertiefenden Darstellung, die Konzentration auf Exponate mit unmittelbarem Themenbezug und das Angebot verschiedener Blickwinkel auf ein Thema. Inhaltliche Konzentration und Multiperspektivität sollen eine analytische Tiefe in der Erarbeitung und Darstellung der Themen fördern, die auch der singulären Wahrnehmung der Großexponate entgegenwirkt. Der vergleichsweise kurze Zeitraum von knapp 140 Jahren militärischer Luftfahrt ist diesem exemplarischen Ansatz dienlich.

Da jeder Erklärung eine Frage vorausgeht, sollen Fragen durch die Ausstellung leiten und der Rundgang als Antwort(en)reise inszeniert werden, der bewusst verschiedene Perspektiven anbieten wird, um festgefügte Geschichtsbilder zu hinterfragen. Wichtig ist zudem, dass sowohl ,Technik-Afficionados“ als auch an Geschichte Interessierte angesprochen werden. Dabei sollen erstere damit konfrontiert werden, dass Technologie nicht isoliert betrachtet werden kann, und denjenigen, die zum Beispiel eher an politischen, kulturellen und sozialen Fragestellungen interessiert sind, soll vermittelt werden, wie eng militärtechnologische Entwicklungen mit eben solchen Aspekten zusammenhängen.

Der Fokus der Ausstellung wird zwar auf Deutschland liegen, doch der Blick kann schon deswegen nicht national begrenzt werden, da militärhistorischen Themen grenzüberschreitende Aspekte eigen sind, wenn es um Krieg und militärische Bündnisse bis hin zu sicherheitspolitischen Fragestellungen geht. 
Im Sinne der gewünschten Multiperspektivität soll hier auch ein komparativer Ansatz zum Zuge kommen, der Unterschiede und Ungleichzeitigkeiten von Entwicklungen in den Blick nimmt und bei einzelnen Themen die enge kuratorische Zusammenarbeit mit Kolleg/innen aus anderen Ländern suchen wird.

Das bisher entworfene inhaltliche Konzept setzt auf einen themenbasierten Ansatz mit Konzentration auf exemplarische Fallbeispiele, die in eine Orientierung gebende Epochenstruktur mit thematisch definierten Epochenbegrenzungen eingebunden werden. Letztere werden durch politisch und technologisch umwälzende Veränderungen markiert, die grundlegende Konsequenzen für den Einsatz von Luftstreitkräften als politisches Mittel nach sich gezogen haben. Eine wichtige Zäsur stellt beispielsweise die Entwicklung und die Einsatzfähigkeit von Nuklearwaffen 1945 dar. Die Drohung mit der Atombombe wurde zu dem politischen Macht- und Druckmittel in der Zeit des Kalten Krieges und der Blockkonfrontation.

Die derzeit noch nicht abschließend festgelegten ca. 15 bis 20 Einzelthemen $^{3}$ sollen aus den drei Perspektiven Politik, Potenzial und Einsatz beleuchtet werden. Sie stehen in engem Zusammenhang mit dem zukünftigen Kernthema des Museums: der Erklärung der Luftstreitkräfte als ein politisches Instrument in historischer Perspektive. Zudem geht es immer auch darum, den Menschen in seinen verschiedenen Rollen und in seinem Bezug zum jeweiligen politischen System in den Mittelpunkt zu stellen. Die Perspektive ,Politik' beinhaltet die politisch-gesellschaftlichen Rahmenbedingungen und Zielsetzungen, die einen Einsatz in der jeweiligen Epoche konditionierten. Dazu gehören Regierungen und weitere Machtinstrumente der jeweiligen Staaten, ferner Öffentlichkeit, Medien, gesellschaftliche Initiativen, Rüstungsindustrie etc. Mit der Perspektive ,Potenzial ' ist der Blick auf die Sach- und Personalmittel gemeint; dazu gehören auch die Technologien, die im Bereich der militärischen Luftfahrt für die Einsätze in den jeweiligen Epochen zur Verfügung standen. Aus der Perspektive ,Einsatz' soll erörtert werden, in welcher Form diese Potenziale in den jeweiligen Epochen eingesetzt wurden. Von der „erklärenden Trias" dieser drei Perspektiven erwarten wir Antworten, die genau auf die Leitfrage zielen: In welchem Kontext sind Luftstreitkräfte legitimes

3 Derzeit diskutiert werden u.a. die folgenden Oberthemen, für deren Darstellung einzelne Fallbeispiele zu definieren sind: Genese von Luftkriegsdoktrinen; Luftaufklärung als Katalysator für die Entwicklung von Luftstreitkräften; Herausbildung eines militärisch nutzbaren Fähigkeitsspektrums und Modernisierungseffekte im Ersten Weltkrieg, Kriegseinsätze als Testfeld von Luftkriegsdoktrinen (z.B. Spanischer Bürgerkrieg); Funktion von Luftstreitkräften bei der Etablierung diktatorischer Systeme im Europa der 1920er und 1930er Jahre; die deutsche Luftwaffe als Mittel zur Expansion des Machtbereichs im Zweiten Weltkrieg; Hintergründe des strategischen Bombenkrieges in Europa 1940-1945; deutsche Rezeption konventioneller Luftkriegskonzeptionen im Kalten Krieg (u.a. Korea 1950-1953); Nuklearstrategien im Spannungsfeld von Perzeption, Abschreckung und Eskalation, Deutschland und die nukleare Teilhabe; Auslandseinsätze der Luftwaffe der Bundeswehr nach 1990 als Ausdruck politischen Gestaltungswillens (z.B. Allied Force 1999). 


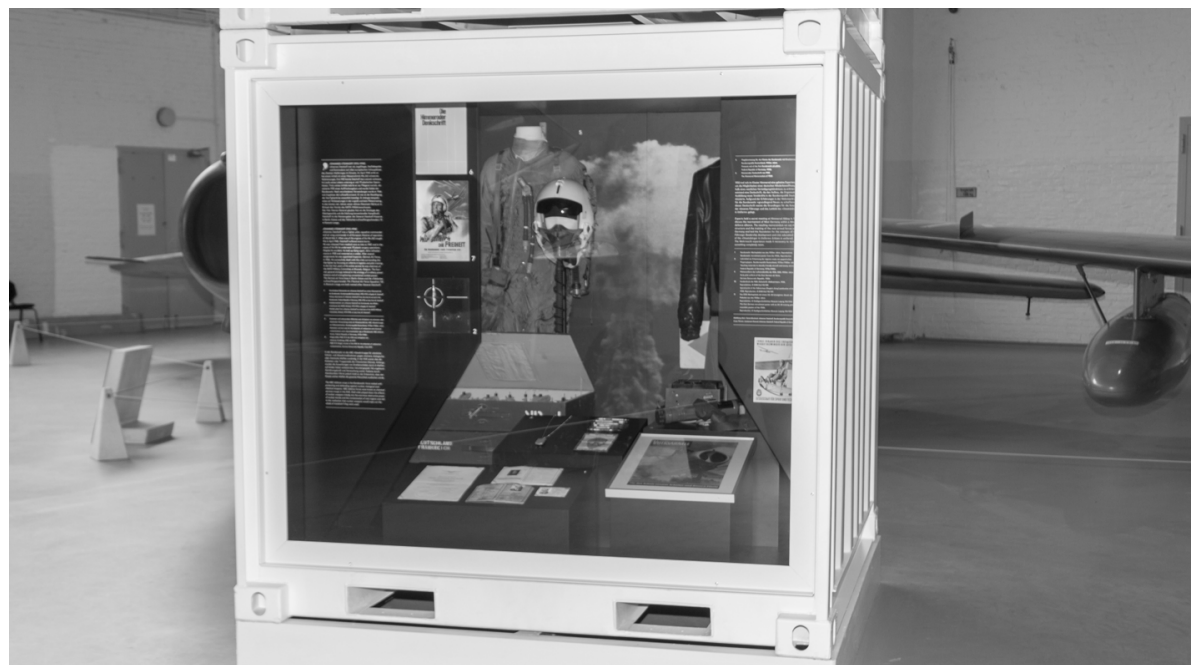

Abb. 2: Inszenierte Vitrine mit Kleinobjekten in einem der Containertürme in der Ausstellung „Zwischenlandung“. (C) Militärhistorisches Museum Flugplatz Berlin-Gatow

und legales Mittel der politischen Interessendurchsetzung eines Staates und in welchem Kontext sind sie es nicht? Die drei Perspektiven können auch als Matrix für die Themen- und die Objektauswahl dienen und aus ihnen soll eine bestimmte Fragenstruktur in Bezug auf die Vermittlung der Einzelthemen beziehungsweise Fallbeispiele abgeleitet werden.

Konkrete Gestaltungsentwürfe werden erst noch erarbeitet, fest steht aber, dass die neue Dauerausstellung möglichst modulhaft aufgebaut werden soll. Auf diese Weise wird die Erneuerung beziehungsweise der Austausch von einzelnen Themen nach einigen Jahren möglich sein, ohne dass die gesamte Ausstellung ab- oder umgebaut werden muss. Sonderausstellungen und weitere Veranstaltungen sollen das Themenspektrum der zukünftigen Dauerausstellung ergänzen.

\section{Kontextualisierung von Großobjekten in der Praxis}

Militärtechnik, insbesondere militärisches Großgerät, übt eine Faszination auf den Betrachter aus. Aufgrund der deutschen Geschichte ist dies - wie oben bereits angesprochen - ein besonders sensibles Thema und Museen, die derartige Exponate ausstellen, sind hier in besonderem Maße gefordert. Dass Militärtechnik fasziniert, polarisiert und provoziert, kann auch als Chance verstanden werden. Ablehnung und Faszination sind starke Emotionen, jenseits von Gleichgültigkeit. Diese „Eigenschaften“ lassen sich nutzen, um Interesse zu wecken und Diskussionen anzuregen.

Das MHM Gatow wendet in seiner aktuellen Dauerausstellung „Zwischenlandung" bereits gezielt Gestaltungsmitteln an, die die Kontextualisierung der Großobjekte fördern und einer unkritischen Faszination entgegenwirken sollen. So werden themenbezogene kleine Objekte wie Dokumente, Plakate, 


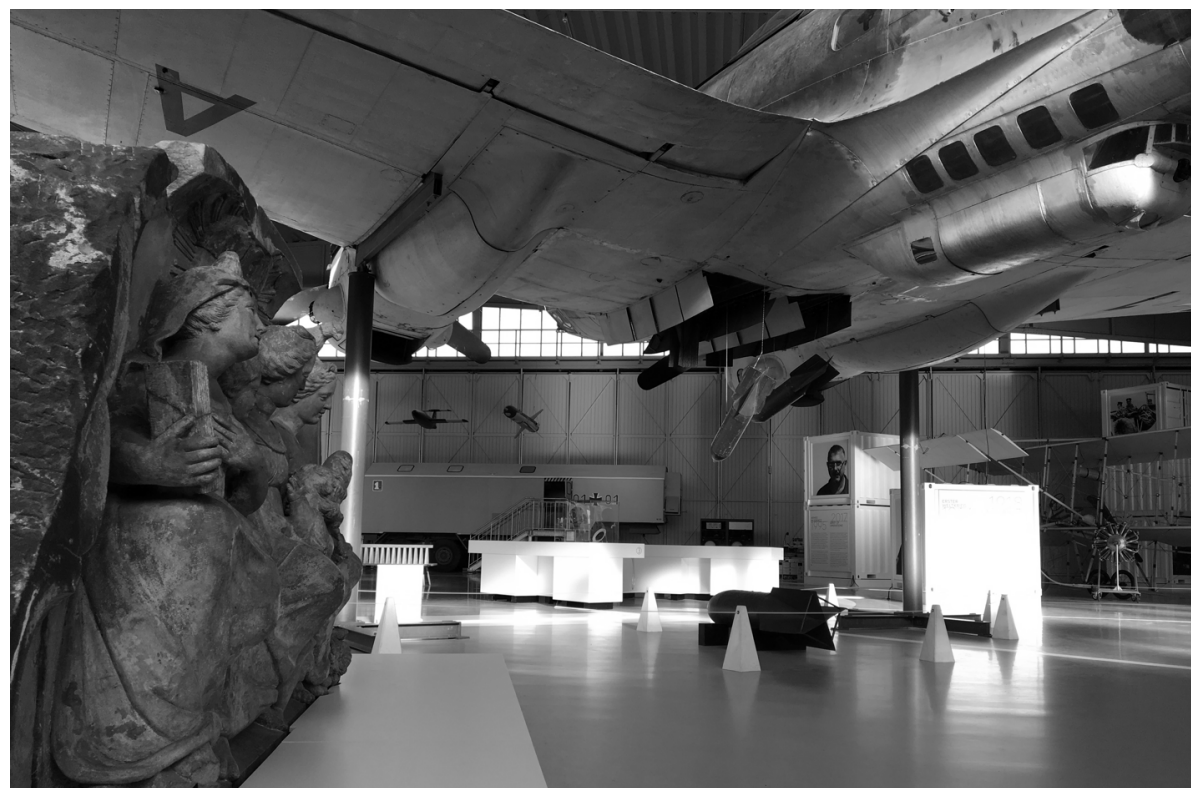

Abb. 3: Giebelrest des Rotterdamer Waisenhauses und Bomber Heinkel He 111. (C) Militärhistorisches Museum Flugplatz Berlin-Gatow

Auszeichnungen etc. zwischen den Großobjekten in inszenierten Vitrinen in Containertürmen präsentiert, die sich im Hangar neben den Luftfahrzeugen behaupten können. Wo immer möglich, wurden diese kleinen Objekte so ausgewählt, dass sie mit persönlichen Geschichten verbunden sind. Auf die Bedeutung des Menschen in seinen verschiedenen Rollen verweisen auch Großporträts von bekannten und unbekannten Protagonisten der militärischen Luftfahrt auf den Containern. Ergänzt wird dieser Ansatz durch Medienstationen mit Zeitzeugenaussagen. Auch wenn es sich hierbei immer um höchst subjektive Äußerungen handelt, ermöglicht das Angebot mehrerer „Stimmen“ zu einem Thema, das noch durch Expertenmeinungen ergänzt werden kann, unterschiedliche Facetten desselben zu erfahren und „öffnet“ die Besucher/ innen auch für schwierige Themen. Außerdem nutzt das MHM Gatow in der derzeitigen Dauerausstellung zur Kontextualisierung Großprojektionen mit authentischem Filmmaterial sowie Objektzusammenstellungen, die beispielsweise Produktionsbedingungen von Waffen oder die Auswirkungen ihres Einsatzes deutlich machen. So werden die Themen „Wunderwaffen“ der Nationalsozialisten, KZ und Zwangsarbeit miteinander verbunden, wird eine während des Zweiten Weltkriegs über Rügen abgeschossene Messerschmitt Bf 108 nicht hochglanzrestauriert, sondern als Wrack konserviert gezeigt und in unmittelbarer Nähe zum Lizenznachbau einer Heinkel He 111 steht ein Trümmerstück vom Giebel des beim deutschen Angriff 1940 mit diesem Bombertyp zerstörten Rotterdamer Waisenhauses. 
Zukünftig soll das Spektrum an Medien und Methoden zur Kontextualisierung noch erweitert werden. $\mathrm{Zu}$ denken ist an gerichtete Soundinstallationen, den Einsatz künstlerischer Mittel und eine verstärkte Besucherbeteiligung, zum Beispiel durch interaktive Stationen zum Thema „Decision Dilemma“, die einen Eindruck von der Komplexität militärischer Handlungsoptionen und Einsatzentscheidungen vermitteln.

Das Ziel ist ein ausgewogener „Mix“ verschiedener Medien und Methoden zur Kontextualisierung. Das ist auch deshalb sinnvoll, weil Studien zeigen, dass Fakten und Informationen nachhaltiger erinnert werden, wenn sie über mehrere „Kanäle“ - beispielsweise lesend und hörend - aufgenommen werden. Zudem gilt es die Waage zu halten zwischen einer dokumentarisch-sachlichen Haltung mit der notwendigen Distanz, um ,verstehen zu können“, und einer sinnlich-emotionalen Ansprache, um den Zugang zu einem schwierigen Themenkomplex zu erleichtern.

Anschrift der Verfasserin: Dr. Doris Müller-Toovey, Leiterin Neukonzeption, Militärhistorisches Museum Flugplatz Berlin-Gatow, Kladower Damm 182188, 14089 Berlin, E-Mail: MHMGatowNeukonzeption@bundeswehr.org 